\title{
Parameter Identification of Li-Po Batteries in Electric Vehicles: a Comparative Study
}

\author{
F. Baronti*, W. Zamboni ${ }^{\S}$, N. Femia ${ }^{\S}$, H. Rahimi-Eichi ${ }^{\ddagger}$, R. Roncella*, S. Rosi ${ }^{*}$ R. Saletti*, and M.-Y. Chow ${ }^{\ddagger}$ \\ ${ }^{*}$ Dip. di Ingegneria dell'Informazione, Università di Pisa, Italy

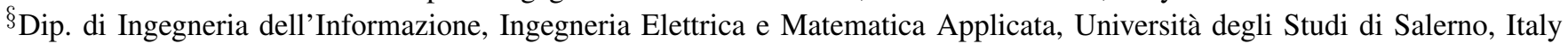 \\ ‡Dept of Electrical and Computer Engineering, North Carolina State University, NC, USA
}

\begin{abstract}
An effective management of the onboard energy storage system is a key point for the development of electric vehicles. This requires the accurate estimation of the battery state over time and in a wide range of operating conditions. The battery state is usually expressed as state-of-charge and state-ofhealth. Its estimation demands an accurate model to represent the static and dynamic behaviour of the battery. Developing such a model requires the online identification of the battery parameters. This paper aims at comparing the performance of two popular system identification techniques, i.e., the Extended Kalman Filter and the classic Least Squares method. A significant contribution of this work is the definition of a benchmark which is representative of the real use of the battery in an electric vehicle. Simulation results show the peculiarities of both methods and their effectiveness.
\end{abstract}

\section{INTRODUCTION}

Electric and Plug-in Hybrid Electric Vehicles (EVs/PHEVs) are one of the most promising technologies to achieve a more sustainable utilisation of energy resources from both economic and environmental points of view. In conjunction with renewable resources, they provide a viable solution to dramatically reduce greenhouse gas emissions. A crucial component of EVs and PHEVs is the energy storage system, which has a huge impact on their cost and performance. On the one hand, Li-ion battery technology, and particularly the Li-Polymer (Li-Po) variant, provides specific and volumetric energy and power densities, charge/discharge efficiency, and lifetime suitable for the implementation of the energy storage system in EVs and PHEVs. On the other hand, a Li-ion cell is very sensitive to overcharge and deep discharge, as well as to be operated outside its safe temperature range. Should these conditions happen, the cell may be permanently damaged. Consequently, an electronic system, called Battery Management System (BMS), is usually employed to protect the battery by measuring the voltage, the temperature, and the current of each cell of the battery. These data feed the algorithms executed by the BMS to evaluate the residual energy stored in the battery and the available power to the load. This information is of paramount importance for an effective management of the battery in the vehicle. It also optimises the battery lifetime, which should be comparable to the life of the vehicle [1].

The main state variables of the battery are the so-called State-of-Charge $(\mathrm{SoC})$ and State-of-Health $(\mathrm{SoH}) . \mathrm{SoC}$ is commonly defined as the ratio of the remaining charge to the cell nominal capacity, where the remaining charge is the residual charge that can be drawn from the cell at room temperature and at a sufficiently low ampere-hour rate [2]. Capacity degradation and internal resistance increase are two indicators of $\mathrm{SoH}$, which are used to predict the Remaining Useful Life (RUL) and End of Life (EOL) of the battery. $S o C$ cannot directly be measured according to its definition without losing the remaining capacity of the cell. Thus, many approaches have been proposed to estimate it [2]-[5].

Coulomb counting, the integration of the battery current over time, is the simplest and most commonly used technique. As it is an open-loop technique based on integration, errors in current measurement due to noise and quantisation accumulate. Thus, large $S o C$ errors can result, calling either for a recalibration or a compensation at regular intervals. This can hardly be feasible in EVs and PHEVs, where the full discharge or charge conditions rarely occur. This also means that the initial value of the integral to calculate $S o C$ may be unknown.

Another simple method to estimate $S o C$ is to use its relationship with the open circuit voltage $V_{\mathrm{OC}}$. However, in LiPo batteries $V_{\mathrm{OC}}$ dependence on $S o C$ is quite little in a wide range of $S o C$, so that a good estimate is only obtained with an accurate measurement of $V_{\mathrm{OC}}$. This is possible with the battery in steady state, a condition reached only after a long time (often several minutes or even hours) with no load current. Thus, this approach is unsuitable for real-time $S o C$ estimation, when the battery is continuously charged or discharged at high currents. Cell-impedance measurements might help in resetting or adjusting $S o C$ estimates from integration-based methods. However, contradictory results arise from various studies undertaken to identify the impedance variation of cells/batteries with $S o C$ [6], [7], so that the usefulness of this technique is still unresolved [2]. Alternative approaches, such as artificial neural networks and fuzzy logic principles, based on a black-box battery model that describes the nonlinear relationship between the $S o C$ and measurable battery variables, can produce good $S o C$ estimates with the drawback of heavy computational burden and poor real-time performance [5].

A very effective approach is offered by the use of state estimation methods based on a state-space battery model. Model-based techniques, such as Kalman Filters (KFs) [8], [9] and Mix algorithm [3], [10], bring the advantages of being closed-loop and are suitable for real-time estimation. A 


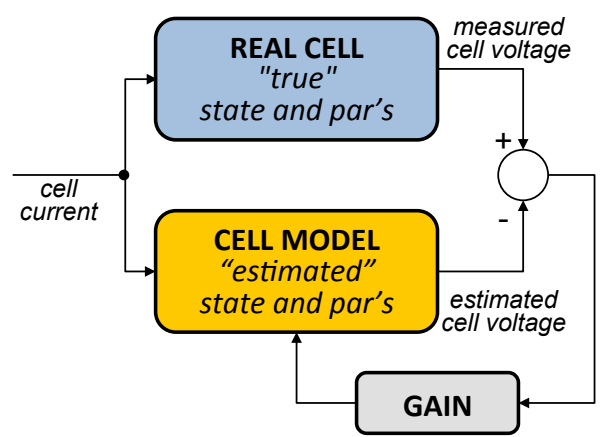

Fig. 1. Schematic block diagram of model-based state-of-charge estimation algorithms.

general view of the basic working principle of these methods is shown in Fig. 1, where the presence of the feedback loop is highlighted. In fact, the output of the Cell model (estimated cell voltage) is compared to the Real cell voltage measured at its terminals (measured cell voltage) and the resulting error is amplified (by the gain block) and fed to the Cell model, where is used to improve the estimation of the model state variables. $S o C$ is then estimated as one of the state variables. It is worth noting that the effectiveness of these techniques strongly depends on how accurately the model describes the underlying cell behaviour. However, the complexity of the usable model is limited by the computational resources typically available on a BMS processor.

An electrical equivalent model seems to be the most suitable. It is relatively simple to compute and accurate enough to predict the cell behaviour at the terminal level [11]. Electrochemical models are capable of accurately reproducing the cell behaviour, at the expense of a higher computational complexity. If compared to a purely mathematical model, an electrical one preserves some significant electrical characteristics of the cell, such as its capacity and internal series resistance, which provides an indication of $\mathrm{SoH}$ [4], [12]. This is important in EVs and PHEVs, where the battery is a key safety-critical component [13].

The parameters of the cell equivalent electrical model need to be tuned to the specific cell being modelled and to its actual operating conditions (cell $S o C$ and temperature) to achieve the desired accuracy. This problem can effectively be tackled by the online identification of the model parameters.

The aim of this work is to compare two popular approaches for parameter identification. The first is the Bayesian estimation with the Extended KF (EKF) [8], [9], i.e., an extension of $\mathrm{KF}$ to the non linear case, where the parameters are considered as additional state variables, and estimated along with $S o C$ in a dual way (Dual EKF). The second approach is the Moving Window Least Squares (MWLS) method applied to the AutoRegressive with eXogenous input (ARX) structure of the equivalent electrical model [14], [15].

\section{ELECTRICAL EQUiVALENT MODEL}

The general representation of a cell equivalent electrical model is shown in Fig. 2 and consists of two parts. The left-

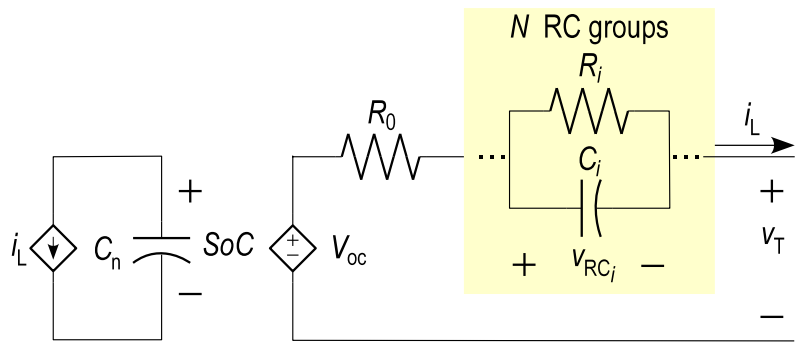

Fig. 2. Electrical equivalent model.

hand side models the charge $Q$ stored in a linear capacitor, which represents the battery capability to accumulate energy. The numerical value of its capacitance $C_{\mathrm{n}}$ is equal to the nominal cell capacity $Q_{\mathrm{n}}$ (expressed in Coulomb) divided by $1 \mathrm{~V}$. $S o C$ can thus be obtained by $Q / Q_{\mathrm{n}}$ and its numerical value is the voltage on the capacitor. On the right-hand side, the cell terminal voltage $v_{\mathrm{T}}$ is obtained by the sum of three terms: $V_{\mathrm{OC}}$, a purely resistive voltage $R_{0} i_{\mathrm{L}}$ (where $i_{\mathrm{L}}$ is the cell terminal current, active sign convention), and the sum of one or more relaxation voltages $v_{\mathrm{RC}_{i}}$ (with time constants $\tau_{i}=R_{i} C_{i}$ ). The time-domain state space model of the cell model, for a generic number $N_{\mathrm{RC}}$ of $\mathrm{RC}$ groups, is the following:

$$
\left\{\begin{array}{l}
S \dot{o} C=-\frac{i_{\mathrm{L}}}{Q_{\mathrm{n}}} \\
\dot{v}_{\mathrm{RC}_{i}}=-\frac{v_{\mathrm{RC}_{i}}}{R_{i} C_{i}}+\frac{i_{\mathrm{L}}}{C_{i}} \\
v_{\mathrm{T}}=V_{\mathrm{OC}}-R_{0} i_{\mathrm{L}}-\sum_{i=1}^{N_{\mathrm{RC}}} v_{\mathrm{RC}_{i}}
\end{array}\right.
$$

Two RC groups (full model) are sufficient to accurately simulate the transient effects in Li-Po cells [11], [16], [17] and good results can also be achieved by only one RC group (reduced model), especially for fast transients. The reduced model is used in this paper as the Cell model of Fig. 1 and its parameters are estimated by both MWLS and EKF identification techniques. The estimates achieved are compared with the Real cell parameters.

The Real cell parameters correspond to the full model (i.e., two RC group model including parameter dependence on state and operating conditions) and have been extracted from experimental offline tests [18] carried out on a $1.5 \mathrm{Ah} \mathrm{Li-Po}$ cell (SLPB723870H manufactured by Kokam), as described in [16]. These parameters have been measured at different cell temperatures (i.e. $10^{\circ} \mathrm{C}, 25^{\circ} \mathrm{C}$ and $35^{\circ} \mathrm{C}$ ) and current levels (i.e., $\mathrm{C} / 2$ and $\mathrm{C}$ during charge and $\mathrm{C} / 2, \mathrm{C}$ and $5 \mathrm{C}$ during discharge, where $\mathrm{C}$ indicates the current that discharges the cell in $1 \mathrm{~h}$ ). They are available in a three-dimensional lookup table (LUT) indexed by the cell $S o C$, temperature, and current. The $V_{\mathrm{OC}}-S o C$ relationship is also expressed by a LUT with different values for each temperature.

If we consider that a higher capacity cell can be thought of as the parallel of smaller ones, the model parameters of a cell 

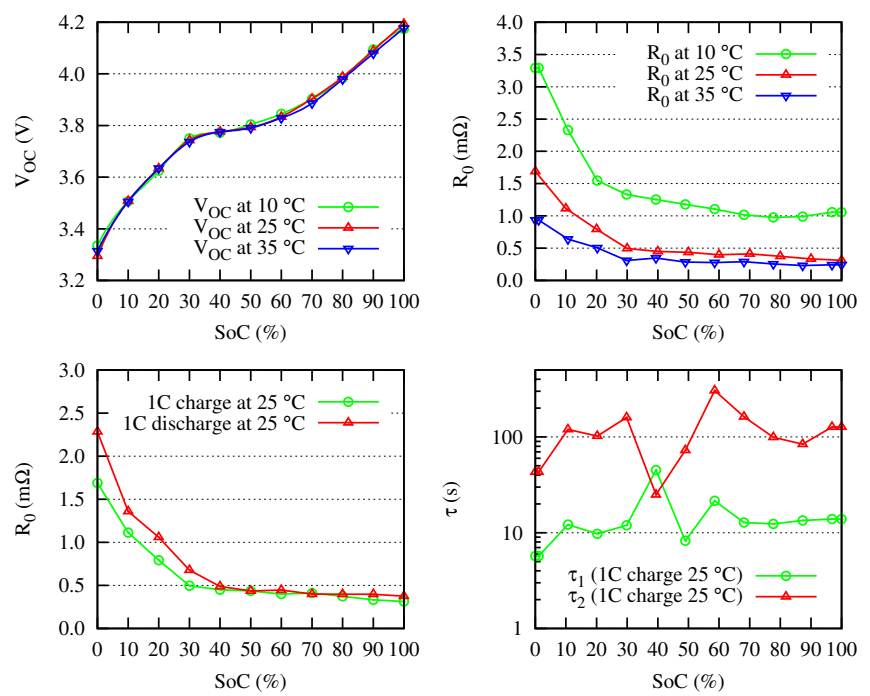

Fig. 3. Parameters of the full model related to a Li-Po cell with a $66.2 \mathrm{Ah}$, as a function of $S o C$. The four diagrams show: open circuit voltage $V_{\mathrm{OC}}$ for various temperatures (top-left), series resistance $R_{0}$ during charge for various temperatures (top-right), series resistance $R_{0}$ for charge and discharge at $1 \mathrm{C}$ (bottom-left), time constants $\tau$ (bottom-right).

with capacity $C^{\prime}$ can be obtained from those extracted from a cell with capacity $C_{0}=1.5 \mathrm{~A} \mathrm{~h}$ by scaling the capacitances of the factor equal to $C^{\prime} / C_{0}$ and the resistances by $C_{0} / C^{\prime}$. Consequently, each time constant $\tau_{i}$ is invariant with the cell capacity.

As an example, Fig. 3 shows the full model parameters of a $66.2 \mathrm{Ah} \mathrm{Li}-\mathrm{Po}$ cell, a size suitable for the battery of an electric vehicle, calculated by scaling the $1.5 \mathrm{Ah}$ data. Fig. 3 clearly shows that the $V_{\mathrm{OC}}-S o C$ relationship is almost temperature independent and, thus, this dependance is neglected in the considered temperature range. On the contrary, the other graphs show that the cell model parameters are strongly dependent on the cell status and the operating conditions. This is evident in the plots showing $R_{0}$ as function of $S o C$ for different values of temperature and current.

Expressing this dependance as a multi-dimensional LUT, as mentioned above, is only feasible for offline simulations, as it requires a time-consuming experimental cell characterisation and should also take into account the parameter variations due to manufacturing tolerances and ageing of the cell. As a consequence, the accurate online identification of the cell model parameters is of paramount importance for enhancing the performance of the $S o C$ estimation algorithms used in an electric vehicle.

\section{COMPARISON METHODOLOGY}

The model parameter identification is strongly affected by the input stimuli, so that it is necessary a testbed representative of the operating condition of the cell, when the battery powers an electric vehicle.

\section{A. Battery current benchmark}

The standard driving cycles used to test passenger cars for assessing the emission levels of the internal combustion engine and fuel economy of the vehicle are a good starting point. The Urban Dynamometer Driving Schedule (UDDS) [19], defined by the U.S. Environmental Protection Agency, is one of them. The New European Driving Cycle is used in Europe and Japan to test passenger cars and light duty trucks. The UDDS cycle is the speed profile shown in Fig. 4, which is usually repeated a given number of times to perform a test.

Given the speed profile of the electric car, we can derive the corresponding current of the battery that powers it. This requires the development of a simulation model of the electric car. The model receives the speed profile as input and generates the electric power at the battery as output. Since our objective is to test different techniques for the online identification of the battery parameters, we used a very simple model to calculate the battery power from the vehicle speed $v$ over time. As shown in (2):

$$
P_{\mathrm{m}}=F v=\left(M \dot{v}+\frac{1}{2} \rho_{\text {air }} S C_{\mathrm{x}} v^{2}+\alpha_{R} M g\right) v
$$

the mechanical power at the wheels $P_{\mathrm{m}}$ is evaluated from the sum of three contributions to the total force $F$ : one term due to the vehicle acceleration and two friction terms due to air resistance (aerodynamics drag) and to rolling resistance (mainly related to tire deformation). Now, we have to turn the mechanical power into the electric power $P_{\mathrm{e}}$ generated by the battery. This is achieved by considering two energy efficiencies: $\eta_{\text {wheel }}$, from the battery to wheels, and $\eta_{\text {reg }}$ in the opposite direction (i.e., during regenerative braking when $\left.P_{\mathrm{m}}<0\right)$.

$$
P_{\mathrm{e}}=\left(\frac{1}{\eta_{\text {wheel }}} \frac{1+\operatorname{sgn}\left(P_{\mathrm{m}}\right)}{2}+\eta_{\text {reg }} \frac{1-\operatorname{sgn}\left(P_{\mathrm{m}}\right)}{2}\right) P_{\mathrm{m}}
$$

The meaning and value of the symbols used in (2) and (3) are defined in Table I. The parameter values have been extracted from a commercial electric car, which is used here as reference electric car.

The last step is to calculate the battery current from $P_{\mathrm{e}}$. To do this, we need to consider the battery of our reference electric car. As shown in the last three rows of Table I, the battery is built up of 96 series-connected Li-Po cells with a nominal capacity of $66.2 \mathrm{~A} \mathrm{~h}$. This results in a battery nominal voltage of $355.2 \mathrm{~V}$ and in a stored energy of $23.5 \mathrm{~kW} \mathrm{~h}$. Let us assume the battery voltage constant and equal to the nominal voltage. The battery current is thus proportional to the electric power: $i_{L}=P_{\mathrm{e}} /\left(N_{\text {cell }} V_{\mathrm{n}}\right)$. This assumption can be justified by noting that the battery voltage approximately varies of $10 \%$, when the $S o C$ is between $20 \%$ and $80 \%$, which is the recommended operating range of a battery in an electric vehicle. Fig. 4 shows the car speed and the battery current in one UDDS cycle. Note the negative values of the battery current during the vehicle deceleration, i.e., when the battery is recharged thanks to regenerative braking.

\section{B. Battery simulation}

The UDDS current calculated above is used as a stimulus for the battery of our reference electric car, in order to identify 
TABLE I

PARAMETERS OF THE REFERENCE ELECTRIC CAR

\begin{tabular}{lll} 
Symbol & Description & Value \\
\hline$M$ & Kerb weight & $1525 \mathrm{~kg}$ \\
$S$ & Frontal area & $2.27 \mathrm{~m}^{2}$ \\
$C_{\mathrm{x}}$ & Drag coefficient & 0.29 \\
$\alpha_{R}$ & Rolling resistance & 0.01 \\
$\rho_{\text {air }}$ & Air density & $1.2 \mathrm{~kg} \mathrm{~m}^{-3}$ \\
$g$ & Gravity acceleration & $9.82 \mathrm{~m} \mathrm{~s}^{-2}$ \\
\hline$\eta_{\mathrm{wheel}}$ & Efficiency from battery to wheel & 0.7 \\
$\eta_{\mathrm{reg}}$ & Efficiency from wheel to battery & 0.5 \\
\hline$N_{\text {cell }}$ & Number of series-connected cells & 96 \\
$C_{\mathrm{n}}$ & Cell nominal capacity & $66.2 \mathrm{~A} \mathrm{~h}$ \\
$V_{\mathrm{n}}$ & Cell nominal voltage & $3.7 \mathrm{~V}$ \\
\hline
\end{tabular}

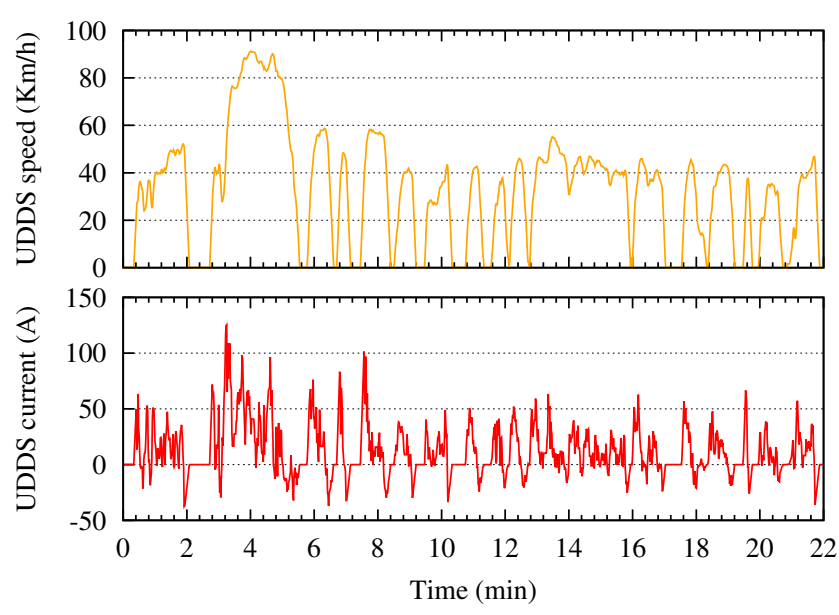

Fig. 4. Urban Dynamometer Driving Schedule.

the parameters of the battery model. As we are considering a virtual electric car, the battery behaviour is simulated by the full model that is capable of reproducing the Real cell behaviour in a very faithful way. We recall here that the model parameters of a scaled size cell were measured in a large range of operating conditions [17], [20]. The model in (1) has been implemented in MATLAB Simulink in the case of $N_{\mathrm{RC}}=2$. The cell capacity is assumed to be constant and equal to the nominal value, while the dependence of $V_{\mathrm{OC}}$ by $S o C$ and of $R_{0}, R_{1}, C_{1}, R_{2}, C_{2}$ by $S o C, i_{\mathrm{L}}$, and temperature is implemented by means of LUTs.

Fig. 5 shows the simulation outputs when the full model is exerted by the UDDS current. In fact, initial $S o C$ of the battery is set to $80 \%$ and the UDDS current is repeated 9 times, so that the battery is discharged down to $20 \%$ of $S o C$ at the end of the test. This is a very reasonable result that reproduces the real battery behaviour in an accurate way. These simulation outputs are used as inputs of the identification methods.

To further extend the analysis and investigate the accuracy of the identification process, we also employed other three Real cell simulations carried out with simplified models that come from keeping the parameters constant and/or disabling the second RC group. Thus, we end up with four different simulations of the Real cell.

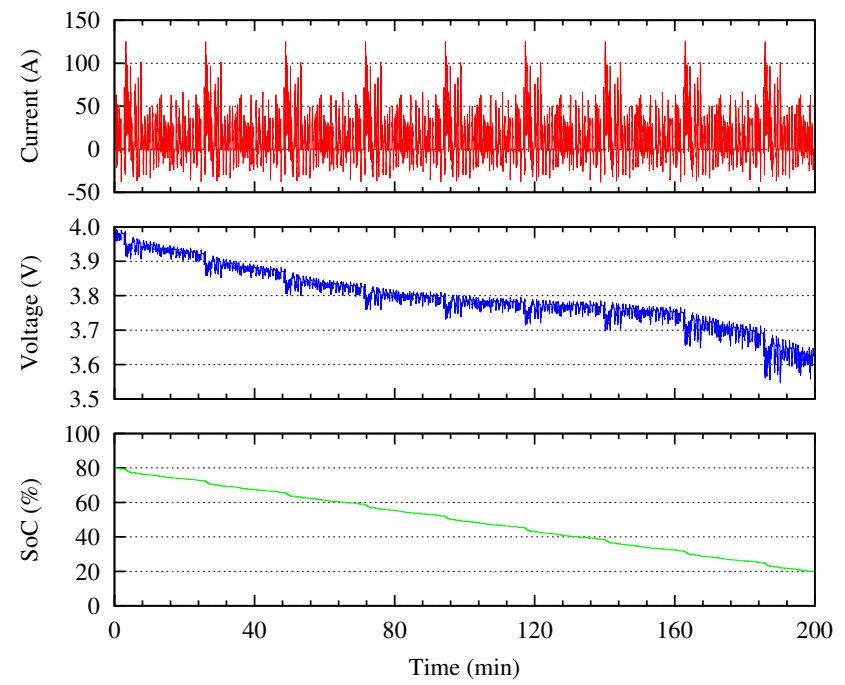

Fig. 5. Battery behaviour in 9 repeated UDDS cycles.

\section{ONLINE PARAMETER IDENTIFICATION}

The ARX and EKF methods will be used to identify the parameters of the reduced model.

\section{A. ARX model least-squares parameter identification}

As described in [14], [15], the first step for using the ARX structure is the linearisation of the reduced model around the time-variant operating point of the cell. As the cell operating point slowly varies over time, we can safely assume that the model parameters remain constant during the identification window. The $V_{\mathrm{OC}}-S o C$ non-linear function is approximated by a piecewise linear curve $V_{\mathrm{OC}}=\alpha_{0}+\alpha_{1} S o C$, where $\alpha_{0}$ and $\alpha_{1}$ change with the operating point. We can now obtain the transfer function from the battery current input to the terminal battery voltage output of the reduced model by the Laplacetransform of the state-space cell model (1) with $N_{\mathrm{RC}}=1$. The input and output variables $(Y(s), U(s))$ are the Laplace transforms of the cell voltage $v_{\mathrm{T}}$ and current $i_{\mathrm{L}}$, respectively.

$$
\frac{Y(s)-\alpha_{0}}{U(s)}=\frac{R_{0} s^{2}+\left(\frac{\alpha_{1}}{Q_{\mathrm{n}}}+\frac{1}{C_{1}}+\frac{R_{0}}{R_{1} C_{1}}\right) s+\frac{\alpha_{1}}{Q_{\mathrm{n}} R_{1} C_{1}}}{\left(s+\frac{1}{R_{1} C_{1}}\right) s}
$$

Applying the bilinear transform, $\left(s \rightarrow \frac{2}{T} \frac{1-z^{-1}}{1+z^{-1}}\right)$, where $T$ is the sampling time, to the continuous transfer function (4), we obtain the discrete transfer function of the system, which is expressed by:

$$
\frac{Y\left(z^{-1}\right)-\alpha_{0}}{U\left(z^{-1}\right)}=\frac{b_{0}+b_{1} z^{-1}+b_{2} z^{-2}}{1+a_{1} z^{-1}+a_{2} z^{-2}}
$$

According to (5) the time-domain relationship between different I/O samples can be written in the following form, which is equivalent to a second order ARX model.

$$
\begin{aligned}
y(k)= & -a_{1} y(k-1)-a_{2} y(k-2)+\alpha_{0}\left(1+a_{1}+a_{2}\right) \\
& +b_{0} u(k)+b_{1} u(k-1)+b_{2} u(k-2)
\end{aligned}
$$




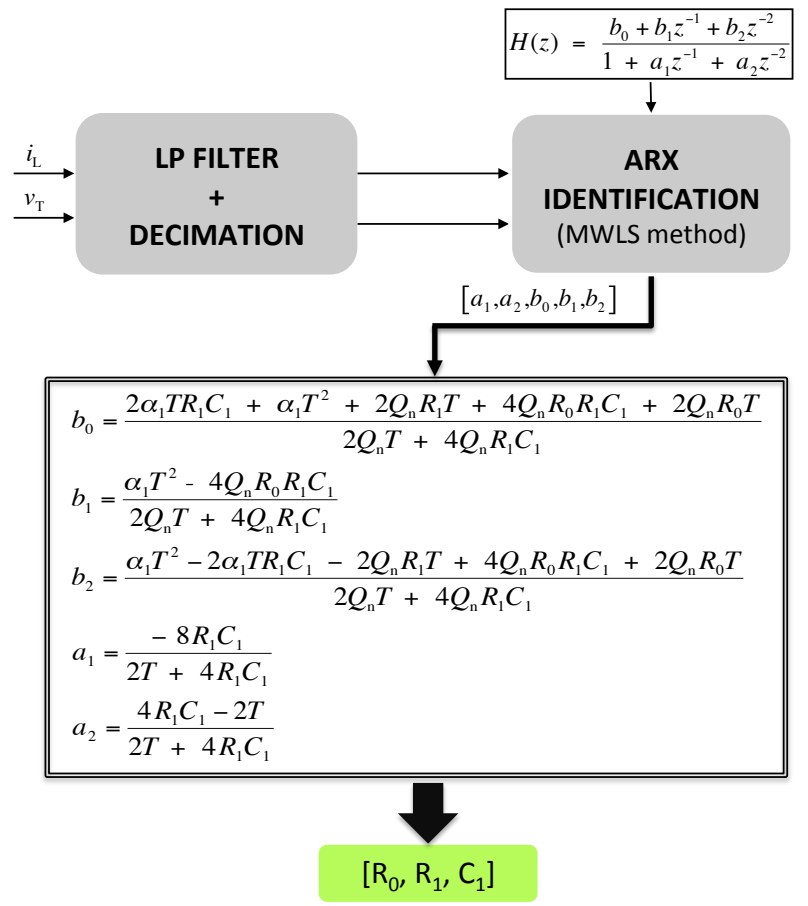

Fig. 6. Moving Window Least Squares parameter identification. The I/O samples are firstly Low-Pass (LP) filtered and decimated before performing the ARX identification.

Using the MWLS technique, we can estimate the parameters $\left[a_{1}, a_{2}, b_{0}, b_{1}, b_{2}\right]$. These are a function of the parameters of the battery. Then, it is possible to obtain the set of parameters $\left[R_{0}, R_{1}, C_{1}\right]$, as shown in Fig. 6. We note that, from the equations shown in Fig. 6, we obtain $1+a_{1}+a_{2}=0$, which means that the value of $\alpha_{0}$ does not affect neither the estimation of the voltage output $y(k)$ nor the model parameters.

\section{B. Bayesian estimation by Kalman filter}

In the Bayesian framework, the discrete evolution of the column vector of parameters $p$ and the corresponding observations (terminal voltage $v_{\mathrm{T}}$ ) are described by the following parameter (7) and measurement (8) equations:

$$
\begin{aligned}
p(k+1) & =p(k)+\chi(k), \\
v_{\mathrm{T}}(k) & =\mathcal{G}\left(x(k), i_{\mathrm{L}}(k), p(k)\right)+\psi(k) .
\end{aligned}
$$

where $k$ is the discrete time, $x$ is the column vector of system state variables (including $S o C$ ), $i_{\mathrm{L}}$ is the terminal current, $p$ is the column vector of parameters, $\chi$ and $\psi$ are the parameter and measurement noise, with zero mean and covariance matrix $\Sigma^{\chi}$ and $\Sigma^{\psi}$, respectively. The measurement operator $\mathcal{G}$ may be linear or non-linear (non linear, in the case of (1)). Equation (7) recasts the problem of parameters estimation in the Bayesian framework: an identity transition operator acts on the parameters, while the parameters dynamics is supposed to be caused by a fictitious Gaussian noise $\chi$. This allows us to dynamically track the parameters, even if the model is not sufficiently detailed (e.g., in the case that $p$ is constant).
In the peculiar case of online estimation, where the tracking of the battery state (in particular: $S o C$ ) is also needed, the model (7)-(8) is further extended to allow the estimation of the battery state and parameters in a dual way [21]. In this case, two parallel interacting systems are assumed to be in force; the first one is given by (7)-(8), and it runs together with the state model:

$$
x(k+1)=\mathcal{F}\left(x(k), i_{\mathrm{L}}(k), p(k)\right)+\xi(k),
$$

being $\mathcal{F}$ the state operator and $\xi$ the state noise. The measurement equation for the state evolution can be the same used for parameters (8), even if the output variable is affected, in principle, by another measurement noise.

Given a sequence of observations for $v_{\mathrm{T}}$, the optimal Bayesian estimator, under mild Markovianity hypotheses, can be recursively calculated into two successive steps [21]. For linear Gaussian statistical models, running two interleaved KFs yields the optimal Bayesian solution [22]. This method turns out to be very effective also in its extended form (EKF), where the presence of non linearities are taken into account [8], [9]. This corresponds to applying a KF to the equations, providing they have been linearised around the actual state and parameter estimates. Implementation details for the dual EKF applied to battery state and parameter estimation can be found in [9] and references therein.

\section{RESULTS}

The simulation framework described in Section III has been employed to compare the performance of the two parameter identification methods described above. With reference to Fig. 1, we recall that the Real cell behaviour has been simulated four different times, under the action of the same UDDS current profile. The Cell model to be identified is always the reduced model.

Fig. 7 shows the results of the identification of the Cell model parameters, i.e., $R_{0}, R_{1}$, and $C_{1}\left(\tau_{1}\right.$ is reported instead of $C_{1}$ ). Each of the four figure rows refers to a specific Real cell simulation, as detailed in the relevant caption. The $V_{\mathrm{OC}}-S o C$ relationship at $25^{\circ} \mathrm{C}$ is used in all cases. When only one RC group is active in the Real cell (cases ab), the Cell model coincides with the Real cell, apart from the dependence over time of the parameters. A very good estimation is provided by both methods. It should be observed that EKF overestimates $R_{0}$ while underestimates $R_{1}$ and, after an initial transient, captures $\tau_{1}$. The MWLS seems to be more noisy, particularly when the $V_{\mathrm{OC}}$ strongly varies with $S o C$ and thus also the model parameters do, but it is quicker to follow the parameter variations.

It is worth noting that we obtained a very good identification of the parameters also when the Real cell is simulated with the full model not simplified (two RC groups along with the dependence of the parameters on the cell state and operating conditions). A situation that represents the real behaviour of a battery very well. In particular, a very good estimation of the series resistance $R_{0}$ is achieved with both methods. This result is very valuable, as $R_{0}$ is the parameter that mostly affect the 

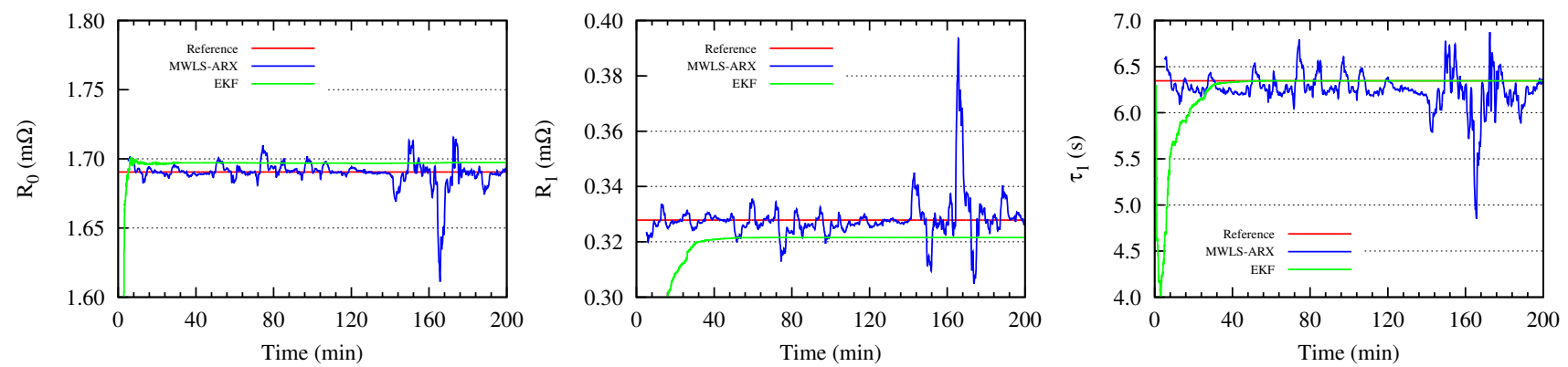

(a) Real cell simulated by the reduced model (1 RC group) with constant parameters
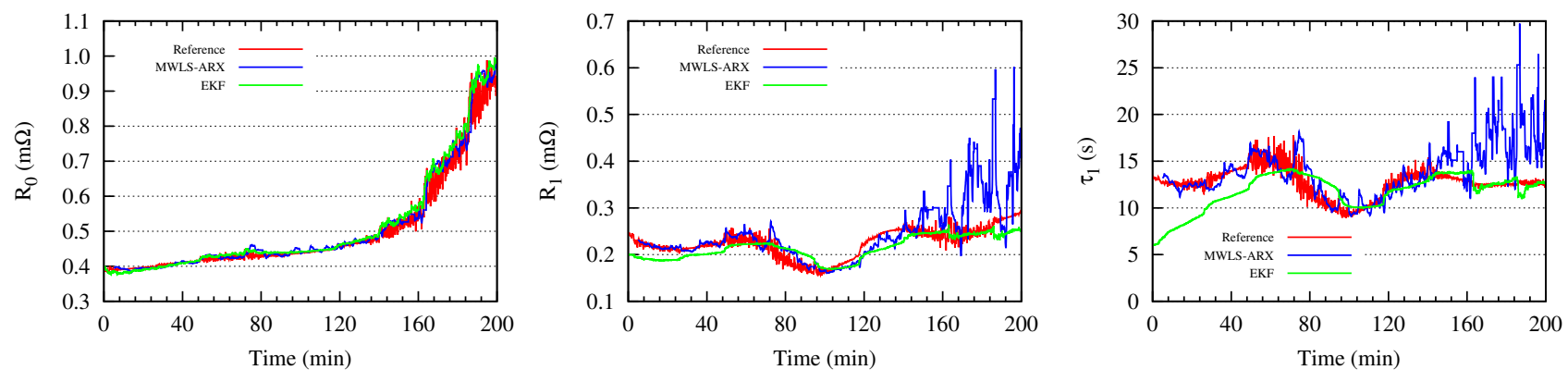

(b) Real cell simulated by the reduced model (1 RC group) with variable parameters
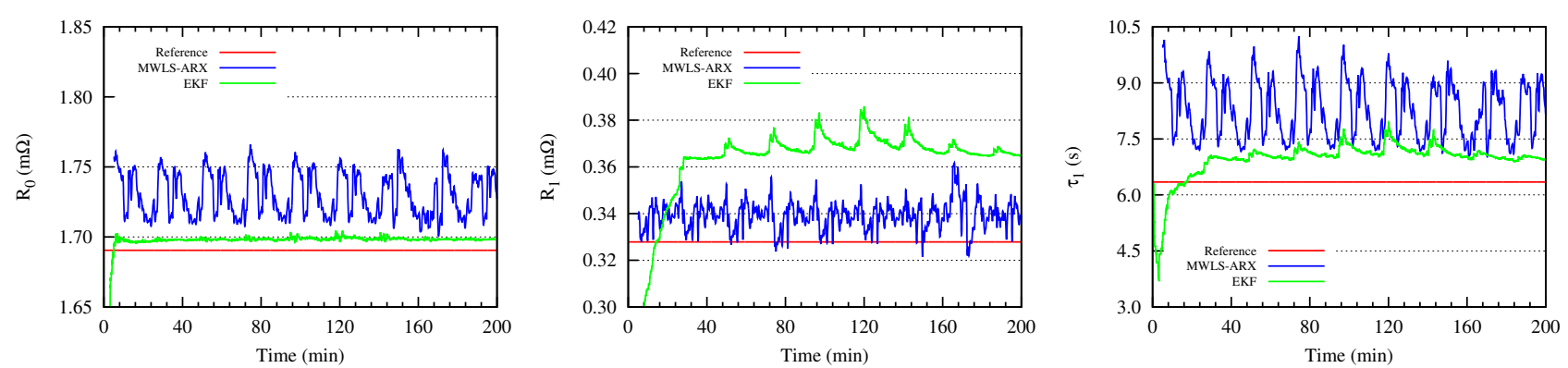

(c) Real cell simulated by the full model (2 RC groups) with constant parameters
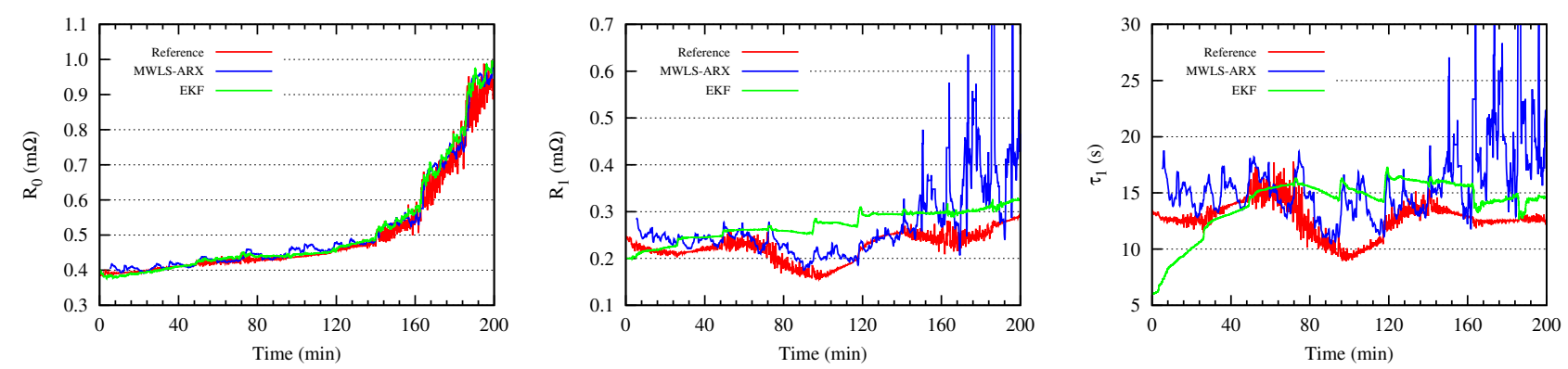

(d) Real cell simulated by the full model (2 RC groups) with variable parameters

Fig. 7. Comparison of the parameter identification results related to four different Real cell simulations.

accuracy of the model and also provides an indication of the $\mathrm{SoH}$.

\section{CONCLUSIONS}

The Extended Kalman Filter and the Moving Window Least Squares method have been applied to the identification of the battery parameters. The battery has been modelled by an equivalent electrical model, which has a low complexity and a few parameters to be identified, but provides a good accuracy in simulating the battery behaviour. A battery current benchmark relevant for an electric vehicle has been obtained starting from a standard driving cycle (Urban Dynamometer Driving Schedule) and used to compare the performance of the parameter identification methods. Both methods have shown good results in identifying the relevant battery model parameters. In particular, MWLS is capable of promptly following 
the parameter variations, but is subject to errors when the nonlinearities of the battery model are significant. On the contrary, EKF can be tuned to be more stable, but this results in a slower tracking of the parameter variations. Future work will focus on investigating the performance of parameter identification and state-of-charge estimation in the presence of noise on battery current and voltage measurements.

\section{ACKNOWLEDGMENT}

This work is partially supported by the U.S. National Science Foundation Award (number: EEC-08212121) and by the University of Salerno FARB funds.

\section{REFERENCES}

[1] K. W. E. Cheng, B. P. Divakar, H. Wu, K. Ding, and H. F. Ho, "Battery-Management System (BMS) and SOC Development for Electrical Vehicles," IEEE Transactions on Vehicular Technology, vol. 60, no. 1, pp. 76-88, Jan. 2011.

[2] S. Piller, M. Perrin, and A. Jossen, "Methods for state-of-charge determination and their applications," Journal of Power Sources, vol. 96, no. 1, pp. 113-120, 2001.

[3] F. Codeca, S. M. Savaresi, and G. Rizzoni, "On battery State of Charge estimation: A new mixed algorithm," in Proc. IEEE Int. Conf. Control Applications CCA 2008, Sep. 2008, pp. 102-107.

[4] H. He, R. Xiong, X. Zhang, F. Sun, and J. Fan, "State-of-charge Estimation of Lithium-ion Battery Using an Adaptive Extended Kalman Filter Based on an Improved Thevenin Model," IEEE Transactions on Vehicular Technology, vol. 60, no. 4, pp. 1461-1469, May 2011.

[5] M. Charkhgard and M. Farrokhi, "State-of-Charge Estimation for Lithium-Ion Batteries Using Neural Networks and EKF," IEEE Transactions on Industrial Electronics, vol. 57, no. 12, pp. 4178-4187, 2010

[6] H. Blanke, O. Bohlen, S. Buller, R. W. D. Doncker, B. Fricke, A. Hammouche, D. Linzen, M. Thele, and D. U. Sauer, "Impedance measurements on leadacid batteries for state-of-charge, state-of-health and cranking capability prognosis in electric and hybrid electric vehicles," Journal of Power Sources, vol. 144, no. 2, pp. 418-425, 2005.

[7] S. Rodrigues, N. Munichandraiah, and A. K. Shukla, "A review of state-of-charge indication of batteries by means of a.c. impedance measurements," Journal of Power Sources, vol. 87, no. 1-2, pp. 12-20, 2000.

[8] G. L. Plett, "Extended Kalman filtering for battery management systems of LiPB-based HEV battery packs: Part 3. State and parameter estimation," Journal of Power Sources, vol. 134, no. 2, pp. 277-292, 2004.
[9] R. Restaino and W. Zamboni, "Comparing particle filter and extended kalman filter for battery State-Of-Charge estimation," in IECON 2012 - 38th Annual Conference on IEEE Industrial Electronics Society. IEEE, Oct. 2012, pp. 4018-4023.

[10] F. Baronti, G. Fantechi, L. Fanucci, E. Leonardi, R. Roncella, R. Saletti, and S. Saponara, "State-of-Charge Estimation Enhancing of Lithium batteries through a Temperature-Dependent Cell Model," in 2011 International Conference on Applied Electronics (AE), Pilsen, 2011, pp. 29-33.

[11] M. Chen and G. Rincon-Mora, "Accurate Electrical Battery Model Capable of Predicting Runtime and I-V Performance," IEEE Transactions on Energy Conversion, vol. 21, no. 2, pp. 504-511, Jun. 2006.

[12] B. Bhangu, P. Bentley, D. Stone, and C. Bingham, "Nonlinear Observers for Predicting State-of-Charge and State-of-Health of LeadAcid Batteries for Hybrid-Electric Vehicles," IEEE Transactions on Vehicular Technology, vol. 54, no. 3, pp. 783-794, May 2005.

[13] E. Meissner and G. Richter, "Battery Monitoring and Electrical Energy Management: Precondition for future vehicle electric power systems," Journal of Power Sources, vol. 116, no. 12, pp. 79-98, 2003.

[14] H. Rahimi-Eichi, F. Baronti, and M.-Y. Chow, "Modeling and online parameter identification of Li-Polymer battery cells for SOC estimation," in 2012 IEEE International Symposium on Industrial Electronics. IEEE, May 2012, pp. 1336-1341.

[15] H. Rahimi-Eichi and M.-Y. Chow, "Adaptive parameter identification and State-of-Charge estimation of lithium-ion batteries," in IECON 2012 - 38th Annual Conference on IEEE Industrial Electronics Society. IEEE, Oct. 2012, pp. 4012-4017.

[16] F. Baronti, G. Fantechi, E. Leonardi, R. Roncella, and R. Saletti, "Enhanced model for Lithium-Polymer cells including temperature effects," in IECON 2010 - 36th Annual Conference on IEEE Industrial Electronics Society. IEEE, Nov. 2010, pp. 2329-2333.

[17] _ , "Effective modeling of temperature effects on lithium polymer cells," in 2010 17th IEEE International Conference on Electronics, Circuits and Systems (ICECS), Athens, Dec. 2010, pp. 990-993.

[18] S. Abu-Sharkh and D. Doerffel, "Rapid test and non-linear model characterisation of solid-state lithium-ion batteries," Journal of Power Sources, vol. 130, no. 1-2, pp. 266-274, 2004.

[19] "Urban Dynamometer Driving Schedule (UDDS)." [Online]. Available: http://www.epa.gov/nvfel/testing/dynamometer.htm

[20] F. Baronti, G. Fantechi, R. Roncella, R. Saletti, and P. Terreni, "Hardware Building Blocks of a Hierarchical Battery Management System for a Fuel Cell HEV," in 2012 38th Annual Conference on IEEE Industrial Electronics Society (IECON), 2012, pp. 1-7.

[21] L. Nelson and E. Stear, "The simultaneous on-line estimation of parameters and states in linear systems," IEEE Transactions on Automatic Control, vol. 21, no. 1, pp. 94-98, Feb. 1976.

[22] R. E. Kalman, "A New Approach to Linear Filtering and Prediction Problems," Transactions of the ASME Journal of Basic Engineering, no. 82 (Series D), pp. 35-45, 1960. 